\title{
AN APPROXIMATION FOR THE POWER FUNCTION OF A SEMI-PARAMETRIC TEST OF FIT
}

\author{
MOHAMMED BOUKILI MAKHOUKHI
}

\begin{abstract}
We consider in this paper goodness of fit tests of the null hypothesis that the underlying d.f. of a sample $F(x)$, belongs to a given family of distribution functions $\mathcal{F}$. We propose a method for deriving approximate values of the power of a weighted Cramér-von Mises type test of goodness of fit. Our method relies on Karhunen-Loève [K.L] expansions on $(0,1)$ for the weighted a Brownian bridges.
\end{abstract}

\section{INTRODUCTION}

In this paper we investigate semi-parametric tests of fit based upon a random sample $X_{1}, X_{2}, \ldots, X_{n}$ with common continuous distribution function $F(x)=$ $\mathbb{P}\left(X_{1} \leq x\right)$. Here $\mathcal{F}=\{G(., \theta): \theta \in \Theta\}$ denotes a family of all distribution function which will be specified later on, and $\Theta$ is some open set in $\mathbb{R}^{k}$.

We seek to test the hypothesis

$$
H_{0}: F(.)=G(., \theta) \in \mathcal{F},
$$

against an alternative which will be specified later on. We will make use of the Cramér-von Mises type statistics of the form

$$
\widehat{W}_{n, \varphi}^{2}:=n \int_{-\infty}^{\infty} \varphi\left(G\left(x, \widehat{\theta}_{n}\right)\right)\left[\mathbb{F}_{n}(x)-G\left(x, \widehat{\theta}_{n}\right)\right]^{2} d G\left(x, \widehat{\theta}_{n}\right),
$$

with $\mathbb{F}_{n}(x)=n^{-1} \sum_{i=1}^{n} \mathbb{I}_{\left\{X_{i} \leq x\right\}}$ denotes the usual empirical distribution function [d.f.] and $\widehat{\theta}_{n}$ is a sequence of estimators of $\theta$ and $\varphi$ is a positive and continuous function on $(0,1)$, fulfilling

$$
\text { (i) } \lim _{t \uparrow 0} t^{2} \varphi(t)=\lim _{t \downarrow 1}(1-t)^{2} \varphi(t)=0
$$$$
\text { (ii) } \int_{0}^{1} t(1-t) \varphi(t)<\infty
$$

Note that, setting $Z_{i}=G\left(X_{i}, \widehat{\theta}_{n}\right)$ for $i=1, \ldots, n$ and letting $\widehat{\mathbb{G}}_{n}(t)$ denotes the empirical d.f. based upon $Z_{1}, \ldots, Z_{n}$ then, we may write, under $\left(H_{0}\right)$,

$$
\widehat{W}_{n, \varphi}^{2}=n \int_{0}^{1} \varphi(t)\left(\widehat{\mathbb{G}}_{n}(t)-t\right)^{2} d t,
$$

with $Z_{1}, \ldots, Z_{n}$ being not independent and identically distributed [i.i.d.] uniform $(0,1)$ r.v's. However, in some important cases the distribution of $Z_{1}, \ldots, Z_{n}$

2000 Mathematics Subject Classification. Primary 62G10, 62F03: Secondary 60J65.

Key words and phrases. Cramér-von Mises tests; Tests of goodness of fit; weak laws; empirical processes; Karhunen-Loève expansions; Gaussian processes; Brownian bridge; Bessel functions. 
doses not depend upon $\theta$, but only on $\mathcal{F}$. In this cases, the distribution of $\widehat{W}_{n, \varphi}^{2}$ is parameter free. This happens if $\mathcal{F}$ is a location scale family and $\widehat{\theta}_{n}$ is an equivalent estimator, a fact noted by David and Johnson [4].

\section{The EMPIRICAL PROCESS WITH ESTIMATED PARAMETERS}

A general study of the weak convergence of the estimated empirical process was carried out by Durbin [6]. We present here an approach to his main results using strong approximations.

Introduce, for each $x \in \mathbb{R}$, the empirical process with estimated parameters

$$
\alpha_{n}\left(x, \widehat{\theta}_{n}\right)=\sqrt{n}\left(\mathbb{F}_{n}(x)-G\left(x, \widehat{\theta}_{n}\right)\right),
$$

where $\widehat{\theta}_{n}$ is a sequence of estimators of $\theta$, and we assume that

$$
\sqrt{n}\left(\widehat{\theta}_{n}-\theta\right)=\frac{1}{\sqrt{n}} \sum_{i=1}^{n} l\left(X_{i}, \theta\right)+o_{\mathbb{P}}(1),
$$

where $l\left(X_{1}, \theta\right)=\left(l_{1}\left(X_{1}, \theta_{1}\right), \ldots, l_{k}\left(X_{1}, \theta_{k}\right)\right)$ is centered function and has finite second moments.

Suppose $F(x)=G(x, \theta) \in \mathcal{F}$ has density $f(x, \theta)=\frac{\partial G}{\partial \theta}(x, \theta)$. Take $\widehat{\theta}_{n}$ as the maximum Likelihood estimator: the maximizer of

$$
m(\theta)=\sum_{i=1}^{n} \log f\left(X_{i}, \theta\right) .
$$

Under adequate regularity conditions $\int \frac{\partial}{\partial \theta} \log f(x, \theta) d G(x, \theta)=0$ and

$\int\left(\frac{\partial}{\partial \theta} \log f(x, \theta)\right)\left(\frac{\partial}{\partial \theta} \log f(x, \theta)\right)^{T} d G(x, \theta)=-\int \frac{\partial^{2}}{\partial \theta^{2}} \log f(x, \theta) d G(x, \theta):=I(\theta)$.

Since

$$
m^{\prime}(\theta)=\sum_{i=1}^{n} \frac{\partial}{\partial \theta} \log f\left(X_{i}, \theta\right) \quad \text { and } \quad m^{\prime \prime}(\theta)=\sum_{i=1}^{n} \frac{\partial}{\partial \theta^{2}} \log f\left(X_{i}, \theta\right),
$$

we obtain, from the Law of Large Number, that $\frac{1}{n} m^{\prime \prime}(\theta) \rightarrow I(\theta)$ almost surely. Now, a Taylor expansion of $m^{\prime}(\theta)$ around $\theta$ gives

$$
\begin{aligned}
\frac{1}{\sqrt{n}}\left(m^{\prime}\left(\widehat{\theta}_{n}\right)-m^{\prime}(\theta)\right) & =\frac{1}{n} m^{\prime \prime}\left(\widehat{\theta}_{n}\right) \sqrt{n}(\theta-\widehat{\theta})+o_{p}(1) \\
& =-I(\theta) \sqrt{n}(\theta-\widehat{\theta})+o_{p}(1),
\end{aligned}
$$

which, taking into account that $m^{\prime}(\widehat{\theta})=0$, gives

$$
\sqrt{n}(\theta-\widehat{\theta})=\frac{1}{\sqrt{n}} \sum_{i=1}^{n} l\left(X_{i}, \theta\right)+o_{p}(1),
$$


with $l(x, \theta)=I(\theta)^{-1} \frac{\partial}{\partial \theta} \log f(x, \theta)$. Clearly $\int l(x, \theta) d G(x, \theta)=0$, while

$$
\int l(x, \theta) l(x, \theta)^{T} d G(x, \theta)=I(\theta)^{-1} I(\theta) I(\theta)^{-1}=I(\theta)^{-1}
$$

To obtain the null asymptotic distribution of $\alpha_{n}\left(x, \widehat{\theta}_{n}\right)$, we assume that $\left(H_{0}\right)$ and (2.4) and write

$$
\begin{aligned}
\alpha_{n}\left(x, \widehat{\theta}_{n}\right) & =\sqrt{n}\left(\mathbb{F}_{n}(x)-G(x, \theta)\right)-\sqrt{n}\left(G\left(x, \widehat{\theta}_{n}\right)-G(x, \theta)\right) \\
& =\alpha_{n}(G(x, \theta))-H(G(x, \theta), \theta)^{T} \int_{0}^{1} L(t, \theta) d \alpha_{n}(t)+o_{\mathbb{P}}(1) \\
& =\widehat{\alpha}_{n}(G(x, \theta))+o_{\mathbb{P}}(1),
\end{aligned}
$$

where $\alpha_{n}($.$) denotes the uniform empirical process, H(t, \theta)=\frac{\partial G}{\partial \theta}\left(G^{-1}(t, \theta), \theta\right)$, $L(t, \theta)=l\left(G^{-1}(t, \theta), \theta\right)$, with $G^{-1}(t, \theta)=\{x: G(x, \theta) \geq t\}$ denoting the quantile function of $X_{1}$, and

$$
\widehat{\alpha}_{n}(t)=\alpha_{n}(t)-H(t, \theta)^{T} \int_{0}^{1} L(s, \theta) d \alpha_{n}(s), \text { for } 0<t<1,
$$

is the uniform estimated empirical process.

2.1. Some notes on stochastic integration. Equation (2.6) suggests that

$$
\widehat{\alpha}_{n}(t) \stackrel{w}{\rightarrow} B(t)-H(t, \theta)^{T} \int_{0}^{1} L(s, \theta) d B(s), \text { as } n \rightarrow \infty,
$$

where $\stackrel{w}{\rightarrow}$ denotes the weak convergence and $B($.$) is a brownian bridge (i.e.,$ a Gaussian process with $B(0)=B(1)=0, \mathbb{E}(B(t))=0, \mathbb{E}(B(s) B(t))=$ $\min (s, t)-s t$ for $s, t \in[0,1])$.

We cannot give $\int_{0}^{1} L(s, \theta) d B(s)$ the meaning of a Stieltjes integral since the trajectories of $B($.$) are not of bounded variation. It is possible, though, to$ make sense of expressions like $\int_{0}^{1} f(s) d B(s)$, with $f \in L^{2}(0,1)$ through the following construction.

Assume first that $f$ is simple : $\left(f(t)=\sum_{i=1}^{n} a_{i} \mathbb{I}_{\left(t_{\left.W<, t_{i-1}\right]}\right.}\right.$, with $a_{i} \in \mathbb{R}$ and $\left.0=t_{0}<t_{1}<\cdots<t_{n}=1\right)$. Then

$$
\int_{0}^{1} f(s) d B(s)=\sum_{i=1}^{n} a_{i}\left(B\left(t_{i}\right)-B\left(t_{i-1}\right)\right):=\sum_{i=1}^{n} a_{i} \triangle B_{i},
$$

where $\triangle B_{i}=B\left(t_{i}\right)-B\left(t_{i-1}\right)$. It can be easily checked that $\mathbb{E}\left(\triangle B_{i}\right)=0$ and $\operatorname{Var}\left(\triangle B_{i}\right)=\triangle t_{i}\left(1-\triangle t_{i}\right) \quad$ and $\quad \operatorname{Cov}\left(\triangle B_{i}, \triangle B_{j}\right)=-\triangle t_{i} \triangle t_{j} \quad$ if $\quad i \neq j$.

The random variable is centered Gaussian with variance 


$$
\begin{aligned}
\sum_{i=1}^{n} a_{i}^{2} \mathbb{V a r}\left(\triangle B_{i}\right)+2 \sum_{1 \leq i<j \leq n} a_{i} a_{j} \operatorname{Cov}\left(\triangle B_{i}, \triangle B_{j}\right) & =\sum_{i=1}^{n} a_{i}^{2} \triangle t_{i}-\sum_{i=1}^{n} \sum_{j=1}^{n} a_{i} a_{j} \triangle t_{i} \triangle t_{j} \\
& =\sum_{i=1}^{n} a_{i}^{2} \triangle t_{i}-\left(\sum_{i=1}^{n} a_{i} \triangle t_{i}\right)^{2} \\
& =\int_{0}^{1} f^{2}(t) d t-\left(\int_{0}^{1} f(t) d t\right)^{2} .
\end{aligned}
$$

Thus, $f \longrightarrow \int_{0}^{1} f(s) d B(s)$ defines an isometry between the subspace of $L^{2}(0,1)$ consisting of centered, simple functions and its range. We can therefore extend the definition to all centered function in $L^{2}(0,1)$. Finally, for a general $f \in L^{2}(0,1)$,

$$
\int_{0}^{1} f(s) d B(s)=f \longrightarrow \int_{0}^{1} \widehat{f}(s) d B(s)
$$

where $\widehat{f}(s)=f(s)-\int_{0}^{1} f(t) d t$. The stochastic integral $\int_{0}^{1} f(s) d B(s)$ is centered, Gaussian random variable with variance

$$
\int_{0}^{1} f^{2}(t) d t-\left(\int_{0}^{1} L(t) d t\right)^{2}
$$

In fact, if $f_{1}, \ldots, f_{k} \in L^{2}(0,1)$, then $\left(\int_{0}^{1} f_{1}(s) d B(s), \ldots, \int_{0}^{1} f_{k}(s) d B(s)\right)$ has a joint centered, Gaussian law and form the isometry defining the integrals we see that

$\operatorname{Cov}\left(\int_{0}^{1} f(s) d B(s), \int_{0}^{1} g(s) d B(s)\right)=\int_{0}^{1} f(s) g(s) d s-\int_{0}^{1} f(s) d s \int_{0}^{1} g(s) d s$.

We can similarly check that

$$
\left(\{B(t)\}_{t \in[0,1]}, \int_{0}^{1} f_{1}(s) d B(s), \ldots, \int_{0}^{1} f_{k}(s) d B(s)\right)
$$

is Gaussian and

$$
\operatorname{Cov}\left(B(t), \int_{0}^{1} f(s) d B(s)\right)=\int_{0}^{t} f(s) d s-t \int_{0}^{t} f(s) d s
$$

(take $g(s)=\mathbb{I}_{(0,1]}(s)$ in $(2.7)$ to check it).

An integration by parts formula. Suppose $h($.$) is simple. Then$

$$
\int_{0}^{1} h(s) d B(s)=\sum_{i=1}^{n} h\left(t_{i}\right)\left(B\left(t_{i}\right)-B\left(t_{i-1}\right)\right)=-\sum_{i=0}^{n-1} B\left(t_{i}\right)\left(h\left(t_{i+1}\right)-h\left(t_{i}\right)\right)=-\int_{0}^{1} B(t) d h(t) .
$$


This result can be easily extended to any $h($.$) of bounded variation and con-$ tinuous on $[0,1]$ :

$$
\int_{0}^{1} h(s) d B(s)=-\int_{0}^{1} B(t) d h(t) .
$$

This integration by parts formula can be used to bound the difference between stochastic integrals and the corresponding integrals with respect to the empirical process:

$$
\left|\int_{0}^{1} h(s) d \alpha_{n}(s)-\int_{0}^{1} h(s) d B_{n}(s)\right| \leq \sup _{0 \leq t \leq 1}\left|\alpha_{n}(t)-B_{n}(t)\right| \int_{0}^{1} d|h|(s),
$$

$B_{n}($.$) is a sequence of brownian bridges.$

We can summarize now the above arguments in the following theorem (see, e.g., $[6])$.

Theorem 2.1. Provided $H(t, \theta)$ is continuous on $[0,1]$ and $L(s, \theta)$ is continous and bounded variation on $[0,1]$ we can define, on a sufficiently rich probability space, $\alpha_{n}($.$) and B_{n}($.$) such that$

$$
\sup _{0 \leq t \leq 1}\left|\widehat{\alpha}_{n}(t)-\widehat{B}_{n}(t)\right|=O\left(\frac{\log n}{\sqrt{n}}\right) \text { almost surly [a.s.], }
$$

where $\widehat{B}_{n}(t)=B_{n}(t)-H(t, \theta)^{T} \int_{0}^{1} L(s, \theta) d B_{n}(s)$ is a centered Gaussian process with function covariance

$$
\begin{array}{ll}
\widehat{K}_{\theta}(s, t) & =\min (s, t)-s t-H(t, \theta)^{T} \int_{0}^{s} L(x, \theta) d x-H(s, \theta)^{T} \int_{0}^{t} L(x, \theta) d x \\
(2.8)+H(s, \theta)^{T}\left[\int_{0}^{1} L(x, \theta) L(x, \theta)^{T} d x\right] H(t, \theta) .
\end{array}
$$

Note that this covariance function can be expressed as $s \wedge t-\sum_{j=1}^{k} \phi_{j}(s) \phi_{j}(t)$ for some real functions $\phi_{j}($.$) . A very complete study of the Karhunen-Loève$ expansion of Gaussian processus with this type of covariance function was carried out in [11].

Exemple 1. We consider $\mathcal{F}=\left\{G_{0}\left(\frac{-\mu}{\sigma}\right): \theta=(\mu, \sigma) \in \mathbb{R} \times \mathbb{R}_{+}^{*}\right\}$ is a location scale family $\left(G_{0}(\right.$.$\left.) is a standard distribution function with density g_{0}\right)$. Then

$$
H(t, \theta)=-\frac{1}{\sigma} g_{0}\left(G_{0}^{-1}(t)\right)\left[\begin{array}{c}
1 \\
G_{0}^{-1}(t)
\end{array}\right]
$$


and

$$
I(\theta)=\frac{1}{\sigma^{2}}\left[\begin{array}{cc}
\int \frac{g_{0}^{2}(x)}{g_{0}(x)} d x & \int x \frac{g_{0}^{2}(x)}{g_{0}(x)} d x \\
\int x \frac{g_{0}^{2}(x)}{g_{0}(x)} d x & \int x^{2} \frac{g_{0}^{2}(x)}{g_{0}(x)} d x-1
\end{array}\right]
$$

We can now write

$$
I(\theta)^{-1}=\sigma^{2}\left[\begin{array}{ll}
\sigma_{11} & \sigma_{12} \\
\sigma_{21} & \sigma_{22}
\end{array}\right]
$$

with $\sigma_{i j}$ depending only on $G_{0}$, but not on $\mu$ or $\sigma$ and

$$
\widehat{K}(s, t)=\min (s, t)-s t-\phi_{1}(s) \phi_{1}(t)-\phi_{2}(s) \phi_{2}(t) .
$$

Here

$$
\phi_{1}(t)=-\sqrt{\left(\sigma_{11}-\frac{\sigma_{12}^{2}}{\sigma_{22}}\right)} g_{0}\left(G_{0}^{-1}(t)\right)
$$

and

$$
\phi_{2}(t)=-\frac{\sigma_{12}}{\sqrt{\sigma_{22}}} g_{0}\left(G_{0}^{-1}(t)\right)-\sqrt{\sigma_{22}} g_{0}\left(G_{0}^{-1}(t)\right) G_{0}^{-1}(t) .
$$

If $\mathcal{F}$ is the Gaussian family $G_{0}(x)=\Phi(x), g_{0}(x)=\phi(x), g^{\prime}{ }_{0}(x)=-x \phi(x)$ and

$$
I(\theta)=\frac{1}{\sigma^{2}}\left[\begin{array}{ll}
1 & 0 \\
0 & 2
\end{array}\right]
$$

Hence, $\sigma_{11}=1, \sigma_{22}=\frac{1}{2}, \sigma_{12}=\sigma_{21}=0$ and

$$
\widehat{K}(s, t)=\min (s, t)-s t-\phi\left(\Phi^{-1}(s)\right) \phi\left(\Phi^{-1}(t)\right)-\frac{1}{2} \phi\left(\Phi^{-1}(s)\right) \Phi^{-1}(s) \phi\left(\Phi^{-1}(t)\right) \Phi^{-1}(t) .
$$

In this Gaussian case $L$ is not of bounded variation on $[0,1]$, but the above argument can be modified and still prove that

$$
\begin{gathered}
\left\{\widehat{\alpha}_{n}(t)\right\}_{t} \stackrel{w}{\longrightarrow} \\
\left\{B(t)+\phi\left(\Phi^{-1}(s)\right) \int_{0}^{1}\left(\Phi^{-1}(s)\right) d B(s)+\frac{1}{2} \phi\left(\Phi^{-1}(t)\right) \Phi^{-1}(t) \int_{0}^{1}\left(\Phi^{-1}(s)^{2}-1\right) d B(s)\right\}_{t}
\end{gathered}
$$

as random variable in $D[0,1]$ or $L^{2}[0,1]$.

Theorem 2.1 provided, as an easy corollary, the asymptotic distribution of a variety of $\widehat{W}_{n, \varphi}^{2}$ statistics under the null hypothesis. In fact, Durbin's results also give a valuable tool for studying its asymptotic power because they include too the asymptotic distribution of the estimated empirical process under contiguous alternatives. A survey of results connected to Theorem 2.1 as well as a simple derivation of it based on Skorohod embedding can be founnd in $[10]$. 


\section{Results (Asymptotic POWer of the $\widehat{W}_{n, \varphi}^{2}$ Test OF FIt)}

Assume that (1.1) and (2.4), under the null hypothesis $\left(H_{0}\right)$, the limiting distribution of $\widehat{W}_{n, \varphi}^{2}$ in (1.2) coincides with the distribution of the random variable

$$
\widehat{W}_{\varphi}^{2}:=\int_{0}^{1} \varphi(t) B^{2}(t, \theta) d t
$$

where $B(t, \theta)$ is a Gaussian random process with zero mean and covariance function

$$
K_{\varphi}(s, t)=\sqrt{\varphi(s) \varphi(t)} \widehat{K}_{\theta}(s, t),
$$

where $\widehat{K}_{\theta}(s, t)$ has been described above in (2.8).

We chose the sequence of local alternatives which depend on the parameters $\theta=\left(\theta_{1}, \ldots, \theta_{k}\right)$ given by

$$
H_{a}: F(.)=F^{(n)}(., \theta),
$$

where $F^{(n)}(., \theta)$ is chosen as a proper distribution function such that $F^{(n)}(., \theta) \rightarrow$ $G(., \theta)$, as $n \rightarrow \infty$, and with $R_{n}():.=\sqrt{n}\left(F^{(n)}(., \theta)-G(., \theta)\right) \rightarrow R(., \theta)$ in the mean square, as $n \rightarrow \infty$, and $R(., \theta)$ is known and satisfies the condition $\int_{-\infty}^{+\infty} R(x, \theta) d x<\infty$.

These kinds of alternatives were proposed and discussed, in particular, by Chibisov [2]. Setting $t=G(x, \theta), \delta(t, \theta)=R\left(G^{-1}(t, \theta), \theta\right)$ and assuming that

$$
\int_{0}^{1} \varphi(t) \delta^{2}(t, \theta) d t<\infty
$$

Under $\left(H_{a}\right)$, with $\delta(., \theta)$ satisfies the condition (3.10), the limiting distribution (as $n \rightarrow \infty$ ) of statistic $\widehat{W}_{n, \varphi}^{2}$ coincides (see, e.g., [2]) with the distribution of r.v:

$$
\begin{aligned}
\widehat{W}_{(\delta, \varphi)}^{2} & =\int_{0}^{1} \varphi(t)[B(t, \theta)+\delta(t, \theta)]^{2} d t \\
& =\int_{0}^{1} \varphi(t) B^{2}(t, \theta)+2 \int_{0}^{1} \delta(t, \theta) \varphi(t) B(t, \theta) d t+\int_{0}^{1} \delta(t, \theta) \varphi^{2}(t) .
\end{aligned}
$$

For a fixed parameter $\theta$ and a level of significance $\alpha \in(0,1)$, there is a threshold of confidence $t_{\alpha}:=t_{\alpha}(\theta)$ satisfying the identity

$$
\mathbb{P}\left(\int_{0}^{1} \varphi(t) B^{2}(t, \theta) d t \geq t_{\alpha}\right)=\alpha .
$$

(see, e.g., [5] for a tabulation of numerical values of $t_{\alpha}$ for the particular cases $\varphi(t)=t^{2 \beta}, \beta>-1$, and, $\left.\alpha=0.1,0.05,0.01,0.005,0.001\right)$. 
In the case above, the asymptotic power of the test of fit based upon $\widehat{W}_{n, \varphi}^{2}$, under the sequence of local alternatives specified by $\left(H_{a}\right)$, is specified by

$$
\mathbb{P}\left(\widehat{W}_{(\delta, \varphi)}^{2} \geq t_{\alpha}\right)=\lim _{n \rightarrow \infty} \mathbb{P}\left(\widehat{W}_{n, \varphi}^{2} \geq t_{\alpha} \mid H_{a}\right) .
$$

Recalling the definitions (1.1) of $\varphi,(3.9)$ of $K_{\varphi}(.,$.$) and, (3.12) of t_{\alpha}$, we set

$$
\begin{aligned}
& g(t, \theta):=\sqrt{\varphi(t)} \delta(t, \theta), \quad x:=\frac{t_{\alpha}-\int_{0}^{1} K_{\varphi}(t, t) d t-\int_{0}^{1} \varphi(t) \delta^{2}(t, \theta) d t}{2}, \\
& A:=\int_{0}^{1} K_{\varphi}^{2}(s, s) d s, \quad B:=\int_{0}^{1}\left[\int_{0}^{1} g(s, \theta) K_{\varphi}(s, t) d s\right]^{2} d t \\
& C:=\int_{0}^{1} \int_{0}^{1}\left[\int_{0}^{1} g(\quad u, \theta) K_{\varphi}(s, u) d u \int_{0}^{1} g(v, \theta) K_{\varphi}(s, v) d v\right]^{2} K_{\varphi}(s, t) d s d t \\
& D^{2}:=\int_{0}^{1} \int_{0}^{1} g(s, \theta) K_{\varphi}(s, t) g(t, \theta) d s d t .
\end{aligned}
$$

Let $\phi$ (resp. $\Phi)$ be the probability density (resp. distribution) function of the standard normal $\mathcal{N}(0,1)$ distribution. Namely,

$$
\phi(x)=\frac{1}{\sqrt{2 \pi}} e^{-\frac{x^{2}}{2}} \text { and } \Phi(x)=\int_{-\infty}^{x} f(u) d u .
$$

Then, for calculating the power function defined in (3.13), we have the following theorem. Recall the definitions (3.14)-(3.15) of $x, A, B, C$ and, $D$.

Theorem 3.1. Under the assumptions above, we have

$$
\begin{gathered}
1-\mathbb{P}\left(\widehat{W}_{(\delta, \varphi)}^{2} \geq t_{\alpha}\right) \\
=\Phi\left(\frac{x}{D}\right)+\left\{\frac{A}{2 D^{2}} H_{1}\left(\frac{x}{D}\right)+\frac{B}{2 D^{\frac{3}{2}}} H_{2}\left(\frac{x}{D}\right)+\frac{C}{4 D^{4}} H_{3}\left(\frac{x}{D}\right)+\frac{B^{2}}{8 D^{6}} H_{5}\left(\frac{x}{D}\right)\right\} \phi\left(\frac{x}{D}\right)+\varepsilon(x) .
\end{gathered}
$$

Here $H_{j}($.$) are Hermite polynomial and, \varepsilon_{k}($.$) is a remainder term fulfilling$

$$
\sup _{y}|\varepsilon(y)| \leq \frac{C_{1}}{\left(D^{2}-\frac{B}{\lambda_{1}}\right)^{\frac{3}{2}}},
$$

where $C_{1}$ is a constant and, $\lambda_{1}$ is the first eigenvalue of the Fredholm transformation $h \rightarrow \int_{0}^{1} K_{\varphi}(s,) h.(s) d s$.

Remark 1. 
The following particular cases are of interest. If, we replace $g(., \theta)$ by $\gamma g(., \theta)$ in the alternatives of (3.10) (for some real parameter $\gamma>0$ ), we obtain that

$$
\sup _{y}|\varepsilon(y)|=o\left(\gamma^{-\frac{3}{2}}\right) \quad \text { as } \quad \gamma \rightarrow \infty .
$$

Proof. The proof of this theorem resembles that which was published (in the case non-parametric) in another article (see, e.g.,[1]).

\section{NUMERICAL EXAMPLE}

As an illustration, we will consider approximate calculation of the power of $\widehat{W}_{n, \varphi}^{2}$ test for verifying the hypothesis of normal distribution.

Here, we consider $\mathcal{F}=\left\{\Phi\left(\frac{-\mu}{\sigma}\right):(\mu, \sigma) \in \mathbb{R} \times \mathbb{R}_{+}^{*}\right\}, \theta=(\mu, \sigma), \widehat{\theta}=\left(\bar{X}, S^{2}\right)$ and,

$$
H_{0}: F(y)=G(y, \theta):=\Phi\left(\frac{y-\mu}{\sigma}\right) .
$$

We chose as an alternative,

$$
\left(H_{a}\right): F(y)=F^{(n)}(y, \theta):=\Phi\left(\frac{y-\mu}{\sigma}\right)+\gamma \frac{R\left(\frac{y-\mu}{\sigma}\right)}{\sqrt{n}}+O\left(\frac{1}{n}\right),
$$

where $R(x)=\frac{1}{4 \sqrt{2 \pi}}\left(3 x-x^{3}\right) e^{-\frac{x^{2}}{2}}$ and, $\gamma$ is a real parameter positive. Setting $t=\Phi\left(\frac{y-\mu}{\sigma}\right)$ and, $\delta(t)=R\left(\Phi^{-1}(t)\right)$, we obtain

$$
\begin{gathered}
K_{\varphi}(s, t)=\sqrt{\varphi(s) \varphi(t)} \widehat{K}_{\theta}(s, t) \\
=\sqrt{\varphi(s) \varphi(t)}\left\{\min (s, t)-s t-\left(1+\frac{1}{2} \Phi^{-1}(s) \Phi^{-1}(t)\right) \phi\left(\Phi^{-1}(s)\right) \phi\left(\Phi^{-1}(t)\right)\right\} .
\end{gathered}
$$

According to the preceding theorem, the asymptotic power of the test of fit based upon $W_{n, \varphi}^{2}$, under the sequence of local alternatives specified by $\left(H_{a}\right)$ in the case above, is calculated for various $\gamma$ and $\alpha$. The accompanying table gives values of the power $\beta_{\gamma}=\mathbb{P}\left(\widehat{W}_{(\delta, \varphi)}^{2}>t_{\alpha}\right)$ for $\varphi \equiv 1$.

\begin{tabular}{|c|c|c|c|c|c|}
\hline$\alpha=0.01$ & $\gamma$ & $\beta_{\gamma}$ & $\alpha=0.001$ & $\gamma$ & $\beta_{\gamma}$ \\
\hline & 3 & 0.2 & & 3 & 0.085 \\
& 4 & 0.53 & & 4 & 0.21 \\
& 5 & 0.851 & & 5 & 0.532 \\
& 6 & 0.98 & & 6 & 0.847 \\
\hline
\end{tabular}

Table. Approximate power for the test goodness of fit

The second column gives various values of the parameter $\gamma$. The third as well as last the columns give power values for $\beta_{\gamma}$. They are compared with the exact values obtained by Martynov [8]. 


\section{REFERENCES}

[1] Boukili Makhoukhi.M. (2008) An approximation for the power function of a non-parametric test of fit. Statistics and Probability Letters, Vol.78, Issue 8, pp. 1034-1042

[2] Chibisov, D.M. (1965) On the investigation of the asymptotic power of match criteria in the case of close alternatives. Theory of Probability and its Applications, translation by SIAM, Vol. 10, no.3, pp. 460-478.

[3] Csörgö, M., Csörgö, S., Horvath, L and Mason, D.M. (1986) Weighted empirical and quantile process. Ann. Probab, Vol. 14, pp. 31-85.

[4] David, F.N. and Johnson, N.L. (1948) The probability integral transformation when parameters are estimated from the sample. Biometrika 35, 182-190.

[5] Deheuvels, P. and Martynov, G. (2003) High Dimensional Probability III. Progress in probability, Vol. 55, 57-93.

[6] Durbin, J. (1973) Weak convergence of the sample distribution function when parameters are estimated. Ann. Stat, vol. 1, 279-290.

[7] Del Barrio, E. (2005) Empirical and quantile processes in the asymptotic theory of goodness of fit tests. Bernoulli., Vol. 11, no.1, pp. 131-189.

[8] Martynov, G. V. (1976) Program for calculating distribution functions of quadratic forms: in numerical statistics (Algorithms and Programs). In Russian, Izd-vo MGU, Moscow.

[9] Martynov, G. V. (1976) Calculation of the limit distributions of statistics for tests of normality of $w^{2}$-type. Teor. Verojatnost. $i$ Primenen., 21(1) pp 3-15.

[10] Shorack, G. R. and Wellner, J. A. (1986) Empirical Process With Applications to Statistics. Wiley, New York.

[11] Sukhatme, S. (1972) Fredholm determinant of a positive definite kernel of a special type and its applications. Ann. Math. Statist. 43, 1914-1926.

L.S.T.A, Université Paris VI, 175, Rue du Chevaleret, 75013 Paris, France

E-mail address: boukili@ccr.jussieu.fr 